\title{
Study on Pretreatment of Coking Wastewater
}

\author{
Wei-zhuo Wang \\ Architecture Engineering College, Northeast Dianli University, Jilin, China, 132012
}

\begin{abstract}
Coking wastewater is one of the main waste coal chemical industry produces, which contains many toxic substances. It will cause serious impact to the environment. At present, biochemical treatment, which is used for the treatment of coking wastewater, is the principal method to solve this problem. Since the coking waste water has very low biodegradability for its composition of macromolecular organic compounds, we confirm that the pretreatment has become the key. In this paper, we used Ozonation for pretreatment, analyzed of test results, and obtained desired results which can provide basic parameters for engineering.
\end{abstract}

Keywords-coking Wastewater; ozone; biodegradability.

\section{INTRODUCTION}

With the development of coal chemical industry, a series of environmental problems gradually reflected[1]. Because of Characteristics of coal chemical industry, the coking wastewater contains high COD and ammonia nitrogen, which are very difficult to treat. At the same time, the key problem is how to treat the macromolecular organic compounds, in which phenols are main components[2]. Phenols is always a difficult problem in biological process for its toxicity and refractory. Advanced oxidation process is an effective method for treatment of refractory organic compounds, which can improve the biodegradability of the sewage Significantly[3]. Ozone contact was used in this study, the experimental data are obtained, and we discussed the application of advanced oxidation at high phenol water.

\section{COKING WASTE WATER}

When coal chemical industry takes different coal for raw materials, its wastewater will have significant difference, but the main pollutants of the wastewater are similar. In general, the COD in the coking wastewater can reach $2000 \sim 4000 \mathrm{mg} / \mathrm{L}$, total phenolic concentration stay in the level from $300 \mathrm{mg} / \mathrm{L}$ to $1000 \mathrm{mg} / \mathrm{L}$, the highest concentration of volatile phenol can reach to $300 \mathrm{mg} / \mathrm{L}$, and ammonia nitrogen concentration was $100 \sim 250 \mathrm{mg} / \mathrm{L}^{[4]}$.

\section{ADVANCED OXIDATION PROCESS FOR THE TREATMENT OF SEWAGE}

The advanced oxidation processes(AOPs) developed in twentieth Century 80's, have capability of using high reactive intermediates generated in physical and chemical process, and it can oxidize organic compounds or improve the biodegradability of waste water ${ }^{[5]}$. AOPs have a wide applicable range and strong oxidizing ability.For this characteristic, AOPs are widely used in the treatment of high toxic Refractory organics. Fenton reagent in water treatment both has oxidation and coagulation.
(1) Fenton process decomposition of $\mathrm{H}_{2} \mathrm{O}_{2}$ catalyzed by $\mathrm{Fe}^{2+}$ in acidic condition( $\mathrm{PH} 2 \sim 5$ ), and the generated $\mathrm{Fe}^{3+}$ can be involved in coagulation and removal of organic pollutants during this process. But this method requires low $\mathrm{PH}$ and a large amount of the catalyst. The reaction generates a lot of sludge containing iron which can cause the second pollution and increase the cost of follow-up treatment ${ }^{[6]}$.

(2) Photo Catalytic Oxidation decompose the organic matter by activated oxidant, Using self-made tio2 hydrosol as catalyst. Because the catalyst is expensive and difficult to reclaim, the present study focused on the improvement of it $^{[7]}$.

(3) Ultrasonic oxidation method is transmitting ultrasound $(16 \mathrm{kHz} \sim 1 \mathrm{MHz})$ to the solution, as a result, local high temperature, high pressure and high local concentrations of oxide are generated. So, organic matter can be rapidly degraded. But the technique is tricky, expensive, and ineffective to hydrophilic, difficult volatile organic substance ${ }^{[8]}$.

(4) Ozone oxidation is the removal of organic pollutants in waste water by using oxidation of ozone. Ozone is a strong oxidizing agent, and has very significant effect on deodorization, disinfection of sewage and removal of organic matter. The method is widely used in water treatment with fast reaction speed and mild condition and without secondary pollution ${ }^{[9]}$.

\section{EXPERIMENT}

(1) Experimental water

In the experiment, the coking wastewater were sampled from lurgi gasifier effluent in Harbin Coal Chemical Industry Co. Ltd. The actual coking wastewater used in this test has go though a series of preprocessing steps, as follows: removal of acid and oil, ammonia distillation, dephenolization (extractant:methyl isobutyl ketone and diisopropyl ether).

(2)Experimental procedure

Detection method: COD, Microwave Digestion Method; $\mathrm{BOD}_{5}$, dilution inoculation method; volatile phenol, directive light intensity method of 4-anino-antipyrine.

After aeration to the original water at constant power with ozone, $\mathrm{COD}, \mathrm{BOD}_{5}$, volatile phenol were obtained, and aeration time is respectively $5 \mathrm{~min}, 10 \mathrm{~min}, 15 \mathrm{~min}$, and lay three parallel samples each dosage group. At the same time, the original water took the same detection as for comparison.

(3)Experimental results

The Experimental results are shown as tab.1. 
TABLE I. EXPERIMENTAL RESULTS

\begin{tabular}{|c|c|c|c|c|}
\hline & original water & ozone(5min) & ozone(10min) & Ozone(15min) \\
\hline COD $(\mathrm{mg} / \mathrm{L})$ & 9855 & 9405 & 9505 & 9905 \\
\hline volatile phenol $(\mathrm{mg} / \mathrm{L})$ & 69.4 & 68.3 & 69.7 & 64.7 \\
\hline $\mathrm{BOD}_{5}(\mathrm{mg} / \mathrm{L})$ & 2451.8 & 3513.3 & 3613.3 & 3667.1 \\
\hline $\mathrm{BOD}_{5} / \mathrm{COD}$ & 0.2488 & 0.3736 & 0.3799 & 0.3702 \\
\hline
\end{tabular}

\section{V.DISCUSSION}

Through analyzing the experimental results, we have found that the water samples changes markedly with ozone aeration. COD and BOD5/COD were raised with the change of aeration time.

Tough ozonation, COD raised significantly after the initial decline (as shown in Fig.1), instead of the expected algate decline, it is mainly due to the large molecular organic matter, which could not be detected by Microwave Digestion Method, change into detectable matter by oxidative decomposition.

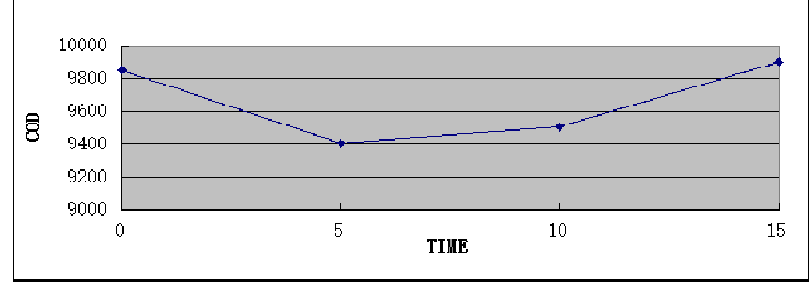

Figure 1. Curves of COD changing with time

BOD5/COD raised significantly and the level goes above 0.3(as shown in Fig.2), this means that the biodegradability of the wastewater is improved by Ozone oxidation. But the effect is no longer obvious after reaction for 5 minutes, this means that the longer reaction time cannot improve the reaction effect further more.

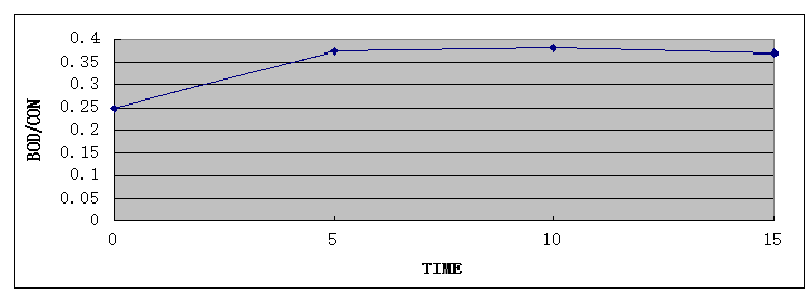

Figure 2. Curves of BOD5/COD changing with time

Volatile phenol, as one of the major phenolic compounds, is not obvious change before and after the reaction(as shown in Fig.3), this means that Volatile phenol is difficult to ozone degradation.

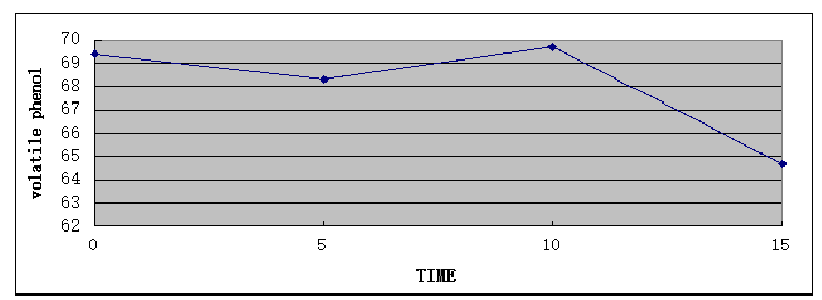

Figure 3. Curves of volatile phenol changing with time

\section{CONCLUSION}

(1) Ozone oxidation can effectively oxidative removal organic compounds, reduce the content of the large molecular organic matter, and provide the foundation for the subsequent reaction without complicated process. At the same time, it is necessary to pay attention to that the reaction effect can not improve with longer reaction time.

(2) Ozone oxidation pretreatment can effectively improve the BOD5/COD level, which means that it can improve biodegradability of the coking waste water, we have strong reason to believe that it is a reliable method to solve the problem of the sewage treatment.

(3) Ozone oxidation play limited roles in treatment volatile phenol, we need special consideration to the volatile phenol in the following advanced treatment.

\section{REFERENCES}

[1] Li H Q, Han H J, Du M A, et al. Removal of phenols, thiocyanate and ammonium from coal gasification wastewater using moving bed biofilm reactor[J]. Bioresource Technology, 2011, 102(7):4667-4673.

[2] Gai H J, Jiang Y B, Qian Y, et al. Conceptual design and retrofitting of the coal-gasification wastewater treatment process[J]. Chemical Engineering Journal, 2008, 138:84-94.

[3] Levee J. Catalytic Oxidation of Toxic Organics in Aqueous Solution[J]. Applied Catalysis, 1990,63:1.

[4] WANG W. Research on Treatment Efficient of Phenolic Compounds in Coal Gasification Wastewater by Enhanced Anaerobic Process[D].Harbin Institute of Technology,2011.

[5] Beitz T, Bechmann W, Mitzner R. Investigations of reactions of selected azaarenes with radicals in water,1. hydroxyl and sulfate radicals [J]. Journal of Physical Chemistry A,1998,102(34):6760 6765.

[6] Perez, M., F. Torrades, X. Domenech, and J. Peral. Fenton and photo-Fenton oxidation of textile effluents. Water Research.2002, 36: 2703-2710.

[7] Senthilnathan J, Philip L. Photo catalyst degradation of lindane under $\mathrm{UV}$ and visble light using N-doped $\mathrm{TiO} 2[\mathrm{~J}]$. Chemical Engineering Journal, 2010, 161(1-2):83-92.

[8] Richard Gedye, Frank Smith, Kennech Westaway, et al. The use of microwave ovens for rapid organic synthesis. Tetrahedron Letters , 1986 , 27(3) :279-282.

[9] Glaze W H' Kang J W,Chapin D H, The chemistry of water and wastewater treatment processes Involving ozone, hydrogen peroxide and ultraviolet radiation. Ozone-Science and Engineering, 1987,9(4): 335-341 